\title{
Editorial
}

Nephrology

\section{Renal Failure in Cirrhosis: Is It Time to Change the Diagnosis and Classification?}

\author{
Suthat Liangpunsakul ${ }^{\mathrm{a}}$ Rajiv Agarwal ${ }^{\mathrm{b}}$ \\ Divisions of a Gastroenterology and Hepatology and ${ }^{\mathrm{b}}$ Nephrology, Department of Medicine, Indiana University \\ School of Medicine, Indianapolis, Ind., USA
}

Renal failure or acute kidney injury (AKI) is one of the most common and severe complications in patients with cirrhosis [1]. These patients have increased propensity for AKI due to the development of hemodynamic abnormalities secondary to portal hypertension and splanchnic vasodilation, which leads to the activation of the renin-angiotensin-aldosterone system. The underlying pathogenesis is believed to be from the increased production of nitric oxide [2]. Once developed, it carries a poor prognosis with high morbidity and mortality $[1,2]$.

The general approach for the diagnosis of AKI in cirrhosis is to dichotomize the etiologies into structural versus functional causes [2]. However, the diagnosis can be very challenging due to the complexity of clinical conditions in these patients. The majority of cases have renal hypoperfusion-related AKI, and the condition improves after adequate volume replacement/expansion. The remaining non-volume responsive cases are classified as having hepatorenal syndrome (HRS), which is further classified into 2 types depending on the evolution of renal failure [3]. HRS type 1 is a rapid worsening of kidney function with the elevation of creatinine from baseline to over $2.5 \mathrm{mg} / \mathrm{dl}$ within 2 weeks. This condition is normally associated with acute alcoholic hepatitis, bacterial infections, and is associated with high short-term (days to weeks) mortality. HRS type 2, on the other hand, is slower in onset and progression. The prognosis is better than that with HRS type 1; however, the overall survival is still poor [3].
At present, the diagnosis of AKI in cirrhosis is based on the presence of serum creatinine $\geq 1.5 \mathrm{mg} / \mathrm{dl}$ (glomerular filtration rate, GFR, $<40 \mathrm{ml} / \mathrm{min}$ ) [4]. The major shortcoming in diagnosis of AKI in patients with cirrhosis is the use of serum creatinine, as the surrogate for GFR. Patients with cirrhosis generally have low muscle mass and inadequate dietary intake. Accordingly, creatininebased equations such as Cockcroft-Gault or Modification of Diet in Renal Disease will overestimate the true GFR [2]. Thus, especially for patients who have low baseline serum creatinine concentration, creatinine-based diagnostics will result in the delay in the identification and thus treatment of AKI.

Because of the shortcoming in creatinine-based diagnosis of AKI in cirrhosis, the new AKI concept and its diagnosis have been proposed by adopting the definition of AKI from the AKI Network (AKIN) instead of using the cutoff creatinine at $1.5 \mathrm{mg} / \mathrm{dl}$ [5]. In this definition, the emphasis of diagnosis of AKI does not rely on structural or functional renal diseases. More importantly, a small increase in serum creatinine $(\geq 0.3 \mathrm{mg} / \mathrm{dl}$ or $\geq 50 \%$ over baseline) is required for the diagnosis of AKI. Once AKI is diagnosed, it can be further subcategorized into three stages according to the magnitude of increase in serum creatinine: stage 1: $150-200 \%$, stage 2 : $>200-$ $300 \%$, stage $3:>300 \%$, or of at least $0.5 \mathrm{mg} / \mathrm{dl}$ in patients with baseline serum creatinine $\geq 4 \mathrm{mg} / \mathrm{dl}$ or renal replacement therapy $[3,5]$. Using the AKIN definition, one

\section{KARGER}

E-Mail karger@karger.com

www.karger.com/ajn
(C) 2013 S. Karger AG, Basel

0250-8095/13/0384-0342\$38.00/0
Suthat Liangpunsakul, MD, MPH

Associate Professor of Medicine

Division of Gastroenterology and Hepatology

550 N. University Blvd, UH 4100, Indianapolis, IN 46202 (USA)

E-Mail sliangpu@iupui.edu 
recent study has found that the risk of mortality is increased with the progression of AKI into the high stages [1]. However, it is important to note that those who had AKI stage 1 , but the total serum creatinine $\leq 1.5 \mathrm{mg} / \mathrm{dl}$ had similar short-term mortality at 90 days to those not having AKI [6].

Regardless of the traditional diagnostic criteria or the new concept of using the AKI definition to identify renal failure, none of these definitions takes the renal hemodynamics such as renal plasma flow (RPF) and GFR into consideration during the diagnosis of renal failure. It is known that patients with Child Class A often have normal renal function despite slight reduction in the RPF [7]. On the other hand, GFR is maintained at normal or low/normal levels by a compensatory increase in filtration fraction, by angiotensin II-induced efferent glomerular arteriole vasoconstriction [7]. The alteration in the RPF, GFR, and filtration fraction is a dynamic process and in fact may coincide with the degree of portal hypertension, and it can be used to stratify the etiologies and stages of renal insufficiency.

In the current issue of the American Journal of $\mathrm{Ne}$ phrology, Mindikoglu and Weir [8], after an excellent topical review, propose a new classification for AKI in cirrhosis. They propose a combination of changes including abnormalities of GFR and RPF to classify kidney dysfunction into 5 different stages from stage 0 to 4 , based on severity. The proposed system is a new dynamic classification in which the status of renal function can change depending on RPF, GFR, and filtration fraction at the time of measurement. In addition, using this approach, the authors indicate and we agree that we will be able to identify unique cases with pre-HRS (cases with reduction in RPF but normal or low/normal GFR) in whom the early intervention and prevention might preclude the progression into HRS [8].

This recent introduction of the concept of renal hemodynamics adds a new facet to the classification of renal dysfunction in cirrhosis. However, its clinical implementation and the feasibility are not yet ready to be primetime. First, GFR and RPF are generally measured using a plasma clearance method, which depends on the determination of specific chemical tracers (inulin and iothalamate for GFR and para-aminohippurate for RPF) being removed from plasma by kidneys $[9,10]$. The measurement requires an experienced laboratory, and it might not be widely available. Second, the classification requires partition of RPF and GFR into different levels depending on the degree of reduction when compared to normal value. However, it is unknown what should be the optimal cutoff for different levels of RPF and GFR. Third, what should be the timing and frequency of the measurement? Patients with cirrhosis are often hospitalized with complicated clinical presentations such as acute variceal bleeding, spontaneous bacterial peritonitis, in conjunction with some degrees of renal insufficiency. Should the measurement be employed at the time of admission or when patient is stabilized? Moreover, the stages of renal disease are dynamic in the newly proposed paradigm; the next question is how often should we measure the renal hemodynamics while patient is hospitalized?

It is unquestionable that we need a sensitive marker to detect early-onset AKI in patients with cirrhosis. The issue of using creatinine is that it is not the indicator to differentiate the types of renal diseases in cirrhosis (structural vs. functional), and therefore it provides no guidance on AKI etiology or as a surrogate for the response to any therapeutic intervention. The use of renal hemodynamics also has its own issues, as outlined above. Recently, several novel urinary biomarkers for structural AKI have been identified. Among them are kidney injury molecule-1, neutrophil gelatinase-associated lipocalin, and liver type fatty acid-binding protein [2]. Increased levels of these markers have been shown to be specific for structural renal injury, which might allow us to differentiate structural from functional renal diseases. Future studies are needed to determine the role of these markers in patients with cirrhosis in the diagnosis of AKI.

In closing, a more accurate classification of AKI in patients with cirrhosis is needed with the goal to detect the condition at the early stage. It would be ideal to use renal hemodynamics in order to stratify patients with different etiologies of renal dysfunction. However, its use has its shortcomings. The recent adaptation of the AKIN criteria to diagnose patients with AKI with cirrhosis seems very promising for the earlier diagnosis of AKI. The use of kidney injury biomarkers is still in its infancy and requires further validation. Clearly, more work is needed in this field in order to explore the new classification to better diagnose AKI in cirrhotic patients.

\section{Acknowledgement}

This study was supported by K08 AA016570 from the NIH/ NIAAA, 1I01CX000361-01 from the Veterans Affairs Research and Administration, and Central Society for Clinical Research Career development award, and Research Support Fund Grant (S.L.). 


\section{References}

1 Belcher JM, Garcia-Tsao G, Sanyal AJ, Bhogal H, Lim JK, Ansari N, Coca SG, Parikh CR: Association of AKI with mortality and complications in hospitalized patients with cirrhosis Hepatology 2013;57:753-762.

2 Belcher JM, Parikh CR, Garcia-Tsao G: Acute kidney injury in patients with cirrhosis: perils and promise. Clin Gastroenterol Hepatol 2013, E-pub ahead of print.

3 Arroyo V: Acute kidney injury (AKI) in cirrhosis: should we change current definition and diagnostic criteria of renal failure in cirrhosis? J Hepatol 2013;59:415-417.
4 Arroyo V, Gines P, Gerbes AL, Dudley FJ, Gentilini P, Laffi G, Reynolds TB, Ring-Larsen $\mathrm{H}$, Scholmerich J: Definition and diagnostic criteria of refractory ascites and hepatorenal syndrome in cirrhosis. International Ascites Club. Hepatology 1996;23:164-176.

5 Kellum JA, Lameire N: Diagnosis, evaluation, and management of acute kidney injury: a KDIGO summary (Part 1). Crit Care 2013;17: 204.

6 Fagundes C, Barreto R, Guevara M, Garcia E, Sola E, Rodriguez E, Graupera I, Ariza X, Pereira G, Alfaro I, Cardenas A, Fernandez J, Poch E, Gines P: A modified acute kidney injury classification for diagnosis and risk stratification of impairment of kidney function in cirrhosis. J Hepatol 2013;59:474-481.
Kew MC, Brunt PW, Varma RR, Hourigan KJ, Williams HS, Sherlock S: Renal and intrarenal blood-flow in cirrhosis of the liver. Lancet 1971;2:504-510.

8 Mindikoglu AL, Weir MR: Current concepts in diagnosis and classification of renal dysfunction in cirrhosis. Am J Nephrol 2013; 38:345-354.

9 Filler G, Yasin A, Medeiros M: Methods of assessing renal function. Pediatr Nephrol 2013, E-pub ahead of print.

10 Mailloux L, Gagnon JA: Measurement of effective renal plasma flow. Prog Nucl Med 1972;2:54-70. 